\title{
Positive and negative results concerning the Gromov-Lawson-Rosenberg conjecture
}

\author{
Michael Joachim* \\ Fachbereich Mathematik \\ Universität Münster \\ Einsteinstr. 62 \\ 48149 Münster
}

\author{
Thomas Schick ${ }^{\dagger}$ \\ Dept. of Mathematics \\ Penn State University \\ 218 McAllister Building \\ University Park, PA 16802
}

Last edited: July 8, 99 — last compiled: August 3, 2018

\begin{abstract}
The Gromov-Lawson-Rosenberg (GLR)-conjecture for a group $\Gamma$ states that a closed spin manifold $M^{n}(n \geq 5)$ with fundamental group $\Gamma$ admits a metric with scal $>0$ if and only if its $C^{*}$-index $\alpha(M) \in K O_{n}\left(C_{r e d}^{*}(\Gamma)\right)$ vanishes. We prove this for groups $\Gamma$ with lowdimensional classifying space and products of such groups with free abelian groups, provided the assembly map for the group $\Gamma$ is (split) injective (and $n$ large enough).

On the other hand, we construct a 5 -dimensional spin manifold $M$ which does not admit a metric with scal $>0$ but has the property that already the image of its $K O$-orientation $p D[M] \in K O_{*}\left(B \pi_{1}(M)\right)$ vanishes. Therefore a corresponding weakened version of the GLRconjecture is wrong.

Last we address non-orientable manifolds. We give a reformulation of the minimal surface method of Schoen and Yau (extended to dimension 8) and introduce a non-orientable (twisted) version of it. We then construct a 5-dimensional manifold whose orientation cover admits a metric of positive scalar curvature and use the latter to show that the manifold itself does not. The manifold also is a counterexample to a twisted analog of the GLR-conjecture because its twisted index vanishes.
\end{abstract}

\footnotetext{
*email: michael.joachim@math.uni-muenster.de www: http://www.math.uni-muenster.de/u/lueck/org/staff/joachim/

${ }^{\dagger}$ e-mail: thomas.schick@math.psu.edu www: http://www.math.psu.edu/schick/ Stay at Penn state funded by the DAAD
} 
MSC-number: 53C20 (global Riemannian Geometry)

\section{Introduction}

This paper studies the question which closed manifolds admit a Riemannian metric with positive scalar curvature. (Throughout the paper, all manifolds are assumed to be smooth.) For connected spin manifolds, the Lichnerowicz method gives a powerful obstruction to this question, the index $\alpha(M)$ which lives in the (real) K-theory of the reduced real $C^{*}$-algebra of the fundamental group.

This invariant just depends on a certain class in a suitably defined bordism group (see below). If the dimension of the spin manifold $M$ is bigger than 4, Gromov and Lawson [6] showed that the existence of a metric with scal $>0$ on $M$ also just depends on this very bordism class. For some time it was conjectured that in these dimensions the vanishing of $\alpha(M)$ is also sufficient for the existence of such a metric. This conjecture is called the Gromov-Lawson-Rosenberg (GLR) conjecture resp. the GLR-conjecture for a given group $\Gamma$ if we restrict the question to manifolds with this fundamental group.

The latter is known to be true for a small class of groups, e.g. for the trivial group [19], cyclic groups of odd order [11, 1.3], $\mathbb{Z} / 2[12,5.3]$ and quaternionic groups [2]. It is also known for the free groups, free abelian groups or the fundamental groups of orientable surfaces [13]. The latter groups all have finite dimensional classifying spaces. The first part of this paper expands this list, in particular dealing with groups with a low dimensional classifying space, and products of such groups with free abelian groups.

More precisely: We show that the statement above is true for $\operatorname{dim}(M) \geq$ $\max \{\operatorname{dim}(B \Gamma)-4,5\}$ if the assembly map $A: K O_{*}(B \Gamma) \rightarrow K O_{*}\left(C_{\text {red }}^{*} \Gamma\right)$ is injective. In particular the GLR-conjecture is true in full generality for hyperbolic groups of dimension at most 9 . In addition we show that if $\operatorname{dim} B \Gamma \leq 5$ and the assembly map is split injective for $\Gamma$ then the GLRconjecture holds for $\Gamma \times \mathbb{Z}^{k} \forall k \in \mathbb{N}$.

The invariant $\alpha$ is defined as follows. Let $\pi$ be the fundamental group of the given closed connected spin manifold $M$. Then $\alpha(M)$ is the image of the bordism class of the classifying map $u: M \rightarrow B \pi$ under a homomorphism $\alpha: \Omega_{n}^{s p i n}(B \pi) \rightarrow K O_{n}\left(C_{r e d}^{*} \pi\right)$, where $K O_{n}\left(C_{r e d}^{*} \pi\right)$ stands for the $n$-th real $K$-theory group of the real reduced $C^{*}$-algebra $C_{r e d}^{*} \pi$, a certain completion

of the group ring $\mathbb{R} \pi$. The homomorphism $\alpha$ is defined through the following 
factorization

$$
\Omega_{n}^{\text {spin }}(B \pi) \stackrel{D}{\rightarrow} k o_{n}(B \pi) \stackrel{p}{\rightarrow} K O_{n}(B \pi) \stackrel{A}{\rightarrow} K O_{n}\left(C_{r e d}^{*} \pi\right),
$$

where $k o_{*}$ is connective real $K$-homology, $K O_{*}$ the periodic real $K$-homology, $D$ is the $k o$-theoretic orientation, $p$ the canonical map between the connective and the periodic theory, and $A$ the assembly map in topological $K$ theory. Note that for torsion free groups, this is the Baum-Connes map, and the Baum-Connes conjecture states that this map is an isomorphism. The homomorphism $\alpha$ also has an analytical interpretation (cf. [11, $\S \S 2-$ $3])$. The image of a class $[N \rightarrow B \pi]$ in $K O_{*}\left(C_{r e d}^{*} \pi\right)$ corresponds to an index of an elliptic differential operator on the manifold $N$. The Lichnerowicz argument shows that this index must be trivial if $N$ admits a metric with positive scalar curvature. I. e. the subgroup $\Omega_{n}^{\text {spin, }+}(B \pi)$ whose elements are bordism classes that can be represented by singular manifolds with positive scalar curvature is contained in the kernel of $\alpha$. However, the counterexample to the GLR-conjecture given by Schick [15] shows that the kernel of $\alpha$ is bigger than $\Omega_{n}^{\text {spin, }+}(B \pi)$ in general. A weaker version of the GLR-conjecture would ask if at least vanishing of $p D[M \stackrel{u}{\rightarrow} B \pi]$ would imply that $M$ admits a metric with scal $>0$. In the second part of this paper we show that this weakened version of the GLR-conjecture fails as well.

If $M$ is not spin, it does not give rise to an element in $\Omega_{*}^{\text {spin }}(B \pi)$ and we cannot apply the above theory. However, there is a twisted version of all this which can be applied whenever the universal covering of $M$ is spin. The twist is essentially decoded by two cohomology classes $u_{1} \in H^{1}(B \pi, \mathbb{Z} / 2)$ and $u_{2} \in$ $H^{2}(B \pi, \mathbb{Z} / 2)$. These classes have to pull back to the first and second StiefelWhitney class of $M$, respectively. We then get an obstruction to positive scalar curvature which lives in $K O_{n}\left(C_{\text {red }}^{*}(\gamma(M))\right.$, the $n$-th real $K$-theory group of a certain $C^{*}$-algebra $C_{\text {red }}^{*}(\gamma(M))$ which is (up to isomorphism) determined by the triple $\left(\pi, u_{1}, u_{2}\right)$. In the third part of the paper we show that the twisted version of the (unstable) GLR-conjecture fails as badly as the untwisted one. The example we give is a non-orientable manifold which does not admit a metric with scal $>0$ but whose orientation cover actually does have such a metric. To show that our manifold indeed is a counterexample, we use a twisted version of the minimal surface method of Schoen and Yau, which relies on geometric measure theory with twists. 


\section{The Gromov-Lawson-Rosenberg conjecture for finite dimensional groups}

2.1. Theorem. Suppose $\Gamma$ is a discrete group and $B \Gamma$ is $N$-dimensional. Suppose $n \geq \max (N-4,5)$ and the assembly map

$$
A: K O_{n}(B \Gamma) \rightarrow K O_{n}\left(C_{r e d}^{*} \Gamma\right)
$$

is injective. Let $M^{n}$ be an $n$-dimensional connected closed spin manifold with $\pi_{1}(M) \cong \Gamma$. Then $M$ admits a metric with positive scalar curvature if and only if its analytical index vanishes, i.e. $\alpha(M)=A p D(M)=0 \in$ $K O_{n}\left(C_{r e d}^{*} \Gamma\right)$.

2.2. Remark. For torsion free groups, injectivity of $A$ is part of the BaumConnes conjecture. ${ }^{1}$ It is known for large classes of discrete groups, e.g. for amenable groups, word-hyperbolic groups, groups of finite asymptotic dimension and all discrete subgroups of Lie groups (cf. Higson [7]). Higson formulates this results for complex $K$-theory. He uses as basic ingredient of his proof that the (complex) Baum-Connes conjecture ${ }^{2}$ is true for amenable groupoids. The Baum-Connes conjecture in the complex case implies the Baum-Connes conjecture in the real case. Starting with the real BaumConnes conjecture for amenable groupoids, the descent argument which Higson uses implies the strong Novikov conjecture also in the real case, which is what we need. ${ }^{\ddagger}$

2.3. Convention. If for fixed $\Gamma$ the conclusion of Theorem 2.1 is true for any $M$ as above with $\operatorname{dim} M \geq 5$ then we say that the Gromov-LawsonRosenberg conjecture is true for $\Gamma$.

The proof of the theorem above relies on the following theorem of Stephan Stolz and Rainer Jung [13, 3.7]

2.4. Proposition. Let $M$ be a connected closed spin manifold with $\pi_{1}(M) \cong$ $\Gamma$ and $\operatorname{dim}(M) \geq 5$. If $D(M) \in k o_{*}(B \Gamma)$ vanishes, then $M$ admits a metric with positive scalar curvature.

To apply this, we first point out some relations between spectra and their connective covers. These can easily be verified. For the reader's convenience we give the proofs here.

\footnotetext{
${ }^{1}$ This part is also called the $K O$-theoretic (strong) Novikov conjecture.

${ }^{2}$ The Baum-Connes conjecture states that the Baum-Connes map (which is the assembly map for torsion free groups) is an isomorphism

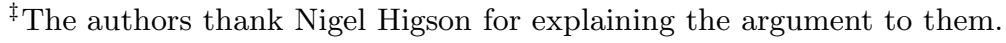


2.5. Lemma. Let $E$ be a spectrum, $f: E\langle k\rangle \rightarrow E$ the $k$-connected cover of $E(k \in \mathbb{Z})$ and let $X$ be a $C W$-complex. Then the induced natural transformation of spectral sequences for the Atiyah-Hirzebruch spectral sequence

$$
f_{p, q}^{n}: E_{p, q}^{n}(E\langle k\rangle(X)) \rightarrow E_{p, q}^{n}(E(X))
$$

is an isomorphism for $q \geq k+n-2$ and surjective for $q \geq k$.

Proof. We prove this by induction on $n$. Since $E_{p, q}^{2}(E(X))=H_{p}\left(X, E_{q}(*)\right)$ and from the definition of $E\langle k\rangle$ the assertion is true for $n=2$. Now suppose the statement is true for some $n \geq 2$. We recall that the $n$-th differentials are a collection of homomorphisms $d_{p, q}^{n}: E_{p, q}^{n} \rightarrow E_{p-n, q+n-1}^{n}$, and let us denote the differentials in the two spectral sequences by $d_{p, q}^{n}\langle k\rangle$ resp. $d_{p, q}^{n}$. If $q \geq k$ then by assumption $f_{p-n, q+n-1}^{n}$ is injective and $f_{p, q}^{n}$ is onto. Naturality then implies that $f_{p, q}^{n}: \operatorname{Ker} d_{p, q}^{n}\langle k\rangle \rightarrow \operatorname{Ker} d_{p, q}^{n}$ is onto, and therefore the same is true for $f_{p, q}^{n+1}$.

If $q \geq k+n-1$ then by assumption $f_{p+n, q-n+1}^{n}$ is onto, and naturality implies that $f_{p, q}^{n}$ restricts to a surjective map $f_{p, q}^{n}: \operatorname{Im} d_{p+n, q-n+1}^{n}\langle k\rangle \rightarrow$ $\operatorname{Im} d_{p+n, q-n+1}^{n}$. Then injectivity of $f_{p, q}^{n+1}$ follows from the injectivity of $f_{p, q}^{n}$, which is given by assumption (since $q \geq k+n-2$ ).

2.6. Lemma. Let $E$ be a spectrum, $f: E\langle k\rangle \rightarrow E$ the $k$-connected cover of $E(k \in \mathbb{Z})$ and let $X$ be a $N$-dimensional $C W$-complex. Then the induced natural transformation on the limit terms of the Atiyah-Hirzebruch spectral sequence

$$
f_{p, q}^{\infty}: E_{p, q}^{\infty}(E\langle k\rangle(X)) \rightarrow E_{p, q}^{\infty}(E(X))
$$

is injective for $p+q \geq k+N-1$ and is an isomorphism for the following indices:

- $q \geq k+N-2$

- $p+q \geq k+N-1$ and $q \geq k$

- $p \geq N+1$.

Consequently, the natural transformation

$$
f_{n}: E\langle k\rangle_{n}(X) \rightarrow E_{n}(X)
$$

is an isomorphism for $n \geq N+k$ and is injective for $n=N+k-1$. 
Proof. The method of proof is identical to the proof of Lemma 2.5, proving a corresponding statement for the stages $E^{k}$, and taking into account that $E_{p, q}^{n}=0$ for $p>N$. One has to note that differentials which map to $E_{0, q}$ are always zero in the Atiyah-Hirzebruch spectral sequence. Because $E^{\infty}=E^{N}$ the statement for the limit term follows.

If $n \geq N+k$ all terms in the filtration of $E\langle k\rangle_{n}(X)$ are mapped isomorphically onto the corresponding terms in the filtration of $E_{n}(X)$. Naturality implies that the induced maps on the homology groups are isomorphisms. Injectivity for $n=N+k-1$ follows from Lemma 2.13.

We want to apply this to $K O$-theory. By definition $k o=K O\langle 0\rangle$. Since $\pi_{-1}(K O)=\pi_{-2}(K O)=\pi_{-3}(K O)=0$ we have $k_{O}=K O\langle-3\rangle$. Hence Lemma 2.6 implies

2.7. Proposition. Suppose $\Gamma$ is a discrete group and $B \Gamma$ is homotopy equivalent to an $N$-dimensional $C W$-complex. Then the natural map

$$
p_{n}: k o_{n}(B \Gamma) \rightarrow K O_{n}(B \Gamma)
$$

is an isomorphism for $n \geq N-3$ and injective for $n=N-4$.

Proof of Theorem 2.1. We only have to show that $p$ and $D$ are injective by $[13,3.8]$. We repeat the argument here. The "only if" part is due to Rosenberg [11]. Suppose now that $A p D(M)=0$. From the assumption about the Novikov conjecture it follows $p D(M)=0$. By Proposition $2.7 p$ is an isomorphism resp. an injection in the dimensions in question. Therefore $D(M)=0$. By the Stolz/Jung result 2.4 the assertion follows.

2.8. Corollary. Let $\Gamma$ be a discrete group with $\operatorname{dim} B \Gamma \leq 9$. Suppose that the injectivity part of the Baum-Connes conjecture holds for $\Gamma$. Then the Gromov-Lawson-Rosenberg conjecture is true for $\Gamma$. In particular it holds for free groups and surface groups and for fundamental groups of complete non-positively curved manifolds of dimension $\leq 9$.

Proof. This is true since the GLR-conjecture is just the assertion of Theorem 2.1 for all $n \geq 5$. For the groups in the list the Novikov conjecture is known to be true by Remark grouplist.

2.9. Remark. To conclude the GLR-conjecture for the free groups or for the fundamental groups of oriented surfaces we do not need to consider the Atiyah-Hirzebruch spectral sequence. Here the injectivity of $p$ follows directly from the fact that in these cases $B \pi$ is stably homotopy equivalent 
to a wedge of spheres and from the suspension isomorphism for homology theories (cf. [13, §3]). This argument can also be used to show the GLRconjecture for all free abelian groups.

2.10. Lemma. If $h_{*}$ is a homology theory then we have canonical isomorphisms $h_{n}\left(S^{1} \times X\right) \cong h_{n}(X) \oplus h_{n-1}(X)$ for spaces $X$ and $n \in \mathbb{Z}$. In particular, if $f: E \rightarrow F$ is a map of spectra then $f_{*}: E_{n}\left(X \times S^{1}\right) \rightarrow F_{n}\left(X \times S^{1}\right)$ is injective if and only if $f_{*}: E_{n}(X) \rightarrow F_{n}(X)$ and $f_{*}: E_{n-1}(X) \rightarrow F_{n-1}(X)$ is injective.

2.11. Proposition. Suppose $G$ is a group with $\operatorname{dim} B G \leq 5$ and the assembly map is injective for $G \times \mathbb{Z}^{k}$. Then the Gromov-Lawson-Rosenberg conjecture holds for $G \times \mathbb{Z}^{k}$.

2.12. Remark. If the assembly map for $G$ is a split injection then the same is true for $G \times \mathbb{Z}^{k}$. This can be seen using Künneth theorems as in Rosenberg's proof of $[11,2.9]$ and the fact that the assembly map is an isomorphism for $\mathbb{Z}^{k}[11,2.6]$. For example, this assumption on $G$ is fulfilled for the groups listed in Remark 2.2.

Proof of Proposition 2.11. We show that $k o_{*}(B G) \rightarrow K O_{*}(B G)$ is an injection for all $* \in \mathbb{Z}$. Then by Lemma 2.10 the same is true for $k o_{*}\left(B G \times \mathbb{Z}^{n}\right) \rightarrow$ $K O_{*}\left(B G \times \mathbb{Z}^{n}\right)$ and the proof of Theorem 2.1 applies.

Again, we study the Atiyah-Hirzebruch spectral sequence for $k o_{*}(B G)$ and $K O_{*}(B G)$. Since $\operatorname{dim} B G \leq 5$ we have $E_{p, q}^{\infty}=E_{p, q}^{6}$. By Lemma 2.5 $E_{p, q}^{\infty}(k o(B G)) \rightarrow E_{p, q}^{\infty}(K O(B G))$ is an isomorphism for $q \geq 0$. Moreover, $E_{p, q}^{\infty}(k o(B G))=0$ for $q<0$. Now apply the following Lemma 2.13.

2.13. Lemma. Let $A$ and $B$ be filtered (graded) groups, filtered by increas-

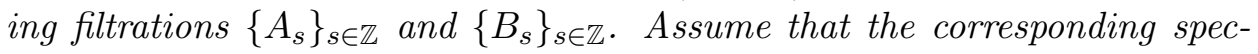
tral sequence converges to $A$ (i.e. $\bigcap_{s \in \mathbb{Z}} A_{s}=\{0\}$ ). If a homomorphism $\varphi: A \rightarrow B$ respects the filtration and induces injections on the $E^{\infty}$-term, then $\varphi$ is injective.

Proof. For any $s \in \mathbb{Z}$ we have a transformation of short exact sequences

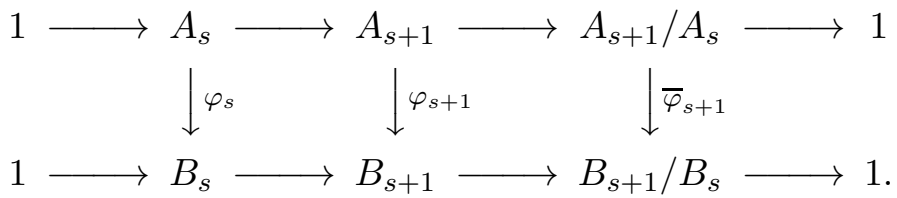


$\bar{\varphi}_{s+1}$ is one of the injections in the $E^{\infty}$-term, hence we have $\operatorname{Ker} \varphi_{s}=$ $\operatorname{Ker} \varphi_{s+1}$. Therefore

$$
\operatorname{Ker} \varphi_{s}=\bigcap_{s^{\prime} \in \mathbb{Z}} \operatorname{Ker} \varphi_{s^{\prime}} \subset \bigcap_{s^{\prime} \in \mathbb{Z}} A_{s^{\prime}}=0 .
$$

I.e. all the $\varphi_{s}$ are injective and therefore so is $\varphi=\lim _{s \rightarrow \infty} \varphi_{s}$.

2.14. Proposition. The GLR-conjecture is true for every group $G$ which fits into an exact sequence $1 \rightarrow \mathbb{Z}^{n} \rightarrow G \rightarrow \mathbb{Z} \rightarrow 1$.

Proof. We study the Atiyah-Hirzebruch spectral sequence for the corresponding fibration of classifying spaces $T^{n} \rightarrow B G \rightarrow S^{1}$. In the case of a fibration over $S^{1}$, we have an action of $\mathbb{Z}$ on the homology of the fiber (induced by the homotopy class of self-maps of the fiber which comes from fiber transport along a generator of $\left.\pi_{1}\left(S^{1}\right)\right)$, and the $E^{2}$-term is given by the invariant and coinvariant elements, respectively.

Note that we can compute $k o_{p}\left(T^{n}\right)$ and $K O_{p}\left(T^{n}\right)$ using the Künneth theorem, and that each of these groups is a product of subgroups which have a given "suspension"-dimension, where an element in $k o_{p-k}(*) \times\left[S^{1}\right]_{i_{1}} \times$ $\ldots\left[S^{1}\right]_{i_{k}}$ has suspension-dimension $k$. Here $\left[S^{1}\right]_{i}$ is a generator of the first homology of the $i$-th factor of $T^{n}$. Since fiber transport gives rise to a map of the first homology of $T^{n}$, the induced map in $K$-theory will preserve the suspension dimension.

The transformation $k o_{p}\left(T^{n}\right) \rightarrow K O_{p}\left(T^{n}\right)$ is exactly the inclusion of those summands with suspension dimension less than $n-p+1$.

We get the following $E^{2}$-terms:

$$
\begin{gathered}
E_{0, q}^{2}(k o)=k o_{q}\left(T^{n}\right) / \mathbb{Z} \stackrel{p}{\rightarrow} K O_{q}\left(T^{n}\right) / \mathbb{Z}=E_{0, q}^{2}(K O) \\
E_{1, q}^{2}(k o)=k o_{q}\left(T^{n}\right)^{\mathbb{Z}} \stackrel{p}{\rightarrow} K O_{q}\left(T^{n}\right)^{\mathbb{Z}}=E_{1, q}^{2}(K O) .
\end{gathered}
$$

All the other groups are trivial. In particular, the $E^{2}$-terms are already the $E^{\infty}$-terms. Since $k o_{*}\left(T^{n}\right)$ is a $\mathbb{Z}$-invariant summand in $K O_{*}\left(T^{n}\right)$, the same is true for the groups of invariant and coinvariant elements, respectively. In particular, the natural transformation induces a (split) injection of the $E^{\infty}$-terms for $k o$ and $K O$. Lemma 2.13 concludes the proof.

2.15. Corollary. If $G$ is the fundamental group of a closed Riemannian manifold whose holonomy is $\mathbb{Z} / p$ for a prime $p$ then the GLR-conjecture is true for $G$.

Proof. Such a group $G$ fits into an exact sequence as in the above proposition (cf. $\left[3,\left(\begin{array}{l}* \\ *\end{array}\right)\right.$ on page 552$]$ ). 


\section{A counterexample to a weak form of the Gromov- Lawson-Rosenberg conjecture}

In this section we present a closed connected spin manifold $M$ with classifying map $M \stackrel{u}{\rightarrow} B G$ such that $p D[M \stackrel{u}{\rightarrow} B G]=0$, but $M$ does not admit a metric with positive scalar curvature. To do this, we start with a closer look at the $K O$-homology of finite groups.

3.1. Proposition. If $G$ is a finite group of odd order then $\widetilde{K O}_{2}(B G)=0$.

Proof. Note the following two facts:

- $\widetilde{K U}_{2}(B G)=0$ by $[5,7.1]$.

- Complexification and forgetting the complex structure yields two natural transformation $c: K O \rightarrow K U$ and $r: K U \rightarrow K O$ whose composition $r c: K O \rightarrow K U \rightarrow K O$ is multiplication by 2 .

These two facts immediately imply that on $\widetilde{K O}_{2}(B G)$ multiplication by two is equal to zero, i.e. $\widetilde{K O}_{2}(B G)$ is a $\mathbb{Z} / 2$-vector space. On the other hand it is well-known that $\widetilde{H}_{*}(G, \mathbb{Z})$ is annihilated by the order of $G$. Hence $\widetilde{H}_{*}(G, \mathbb{Z})$ is odd torsion and multiplication by 2 is an isomorphism on $\widetilde{H}_{*}(G, \mathbb{Z})$. Using the Atiyah-Hirzebruch spectral sequence and the 5-lemma, we see that multiplication by 2 is an isomorphism on the $\mathbb{Z} / 2$-vector space $\widehat{\mathrm{KO}_{2}}(B G)$. Therefore this group must be trivial.

3.2. Proposition. Let $G$ be a discrete group. Then

$$
\Omega_{2}^{\text {spin },+}(B G):=\left\{\begin{array}{c}
\text { bordism classes }[M \rightarrow B G] \in \Omega_{2}^{\text {spin }}(B G), \\
\text { where } M \text { admits a metric with scal }>0
\end{array}\right\}=0 .
$$

Proof. By the Gauss-Bonnet theorem there is only one orientable 2-manifold with positive (scalar) curvature, namely $S^{2}$. Since $\pi_{2}(B G)$ is trivial, up to homotopy only the trivial map from $S^{2}$ to $B G$ exists. Therefore only the trivial element in $\Omega_{2}^{\text {spin }}(B G)$ can be represented by a manifold with positive scalar curvature.

3.4. Proposition. For $G=\mathbb{Z} / 3 \times \mathbb{Z} / 3$, the index map

$$
p D: \Omega_{2}^{s p i n}(B G) \rightarrow K_{2}(B G)
$$

has a non-trivial kernel.

In particular, not every element of this kernel lies in $\Omega_{2}^{\text {spin, }+}(B G)$. 
Proof. It is sufficient to show this for the reduced groups. Since by Proposition $3.1 \widetilde{K O}_{2}(B G)=0$, we only have to show that $\widetilde{\Omega}_{2}^{\text {spin }}(B G)$ is non trivial. This follows immediately from the Atiyah-Hirzebruch spectral sequence since $E_{2,0}^{2} \cong H_{2}(B \mathbb{Z} / 3 \times B \mathbb{Z} / 3, \mathbb{Z}) \neq 0$, and the only possible differential at $(2,0)$ is the $E^{2}$-differential mapping to $E_{0,1}^{2}$. This differential is zero because the coefficients survive to $E^{\infty}$.

3.6. Theorem. There is a 5-dimensional closed connected spin manifold $M$ with $\pi_{1}(M):=\pi \cong \mathbb{Z}^{3} \times \mathbb{Z} / 3 \times \mathbb{Z} / 3$ such that

$$
p D[M]=0 \in K O_{5}(B \pi),
$$

but $M$ does not admit a metric with positive scalar curvature.

The proof, using the minimal surface technique of Schoen and Yau, will be given in the next section.

3.7. Remark. This is a counterexample to one direction of an older conjecture of Gromov and Lawson, namely that positive scalar curvature is equivalent to the vanishing of $p D[M]$. The other direction of the conjecture was disproved by Rosenberg [9]. He then suggested that vanishing of the image $\alpha(M)$ of $p D[M]$ under the assembly map might be sufficient for the existence of a metric with scal $>0$ on $M$. In the following this has become known as the GLR-conjecture.

Dwyer and Stolz have another counterexample like $M$ with the additional very nice property that $\pi_{1}(M)$ is torsion-free. Their computation is based on homotopy theoretic considerations and the Baumslag-Dyer-Heller construction of groups with given homology [1]. The drawback of this approach is that the group they construct is rather artificial.

\section{The minimal surface obstruction to positive scalar curvature}

The following theorem is the differential geometrical backbone for the application of minimal surfaces to the positive scalar curvature problem:

4.1. Theorem. Let $\left(M^{m}, g\right)$ be a Riemannian manifold with scal $>0$, $\operatorname{dim} M=m \geq 3$. If $V$ is a smooth $(m-1)$-dimensional immersed submanifold of $M$ with trivial normal bundle, and if $V$ is a local minimum of the $(m-1)$-volume, then $V$ admits a metric with scal $>0$, too. Note that orientability is not required. 
Proof. [16, Proof of Theorem 5.1] for $m=3$, and [17, Proof of Theorem 1] for $m>3$. The computations there are formulated only for $M$ and $V$ oriented, but they carry over literally to the case where $V$ has trivial normal bundle in $M$.

4.2. Theorem. Suppose $M$ is a closed Riemannian manifold with $\operatorname{dim} M=$ $m \leq 8$. Furthermore let $\alpha \in H^{1}(M, \mathbb{Z})$, and let $\widetilde{o}$ denote the orientation bundle of $M$. Then

$$
x:=\alpha \cap[M] \in H_{m-1}(M, \widetilde{o})
$$

can be represented by an embedded hypersurface $V$ with trivial normal bundle which is a local minimum for $(m-1)$-volume (if $m=8$ with respect to suitable metrics arbitrarily close in $C^{3}$ to the metric we started with).

Note that $V$ also represents $\bar{x} \in H_{m-1}(M, \mathbb{Z} / 2)$, the $\bmod 2$ reduction of $x \in H_{m-1}(M, \widetilde{o})$.

Proof. If $M$ is orientable then we do not have to twist the coefficients and for $m \leq 7$ this is a classical result of geometric measure theory (cf. [8, Chapter 8]). The case $m=8$ follows from the following result of Smale [14]: the set of $C^{k}$-metrics for which the regularity statement holds is open and dense in the set of all $C^{k}$-metrics $(k \geq 3$ and with the usual Banach-space topology). We are only interested in $C^{\infty}$-metrics. But these are dense in the set of $C^{k}$-metrics, which concludes the proof for the orientable case.

More generally, if we work with homology and cohomology with twisted coefficients in the orientation bundle and its dual, respectively, then every submanifold with orientable normal bundle represents a homology class in this theory (being dual to the corresponding differential forms), and there is a Poincaré duality between this homology theory and untwisted integral cohomology. Now one can tediously check that all the basic results of geometric measure theory are true for this theory with twisted coefficients, including the work of Smale. In particular, for each twisted $(m-1)$-cohomology class we find a representing current with minimal volume, and such a minimizing current is a smooth embedded hypersurface with trivial normal bundle if $m \leq 7$, and we can do this for a generic metric if $m=8$. Details of this will be presented in a forthcoming paper.

Remember that if we are given a class $\alpha \in H^{1}(M, \mathbb{Z})$ we may represent it by a map $f: M \rightarrow S^{1}$ being transverse to $1 \in S^{1}$. Then $V=f^{-1}(1) \subset M$ represents $\alpha \cap[M]$ (and conversely, every hypersurface representing $\alpha \cap[M]$ is obtained in this way). Furthermore, if $f^{\prime}: M \rightarrow S^{1}$ is a second map as above 
and $V^{\prime}=f^{\prime-1}(1)$ then $f$ and $f^{\prime}$ are homotopic, and a homotopy $H: f \simeq f^{\prime}$ being transverse to $1 \in S^{1}$ provides a bordism $W=H^{-1}(1): V \sim V^{\prime}$ embedded in $M \times[0,1]$. If $\Omega$ denotes some $B$-bordism theory ${ }^{3}$ and $M$ is equipped with a (normal) $B$-structure then the above procedure provides a class in $\Omega_{m-1}(M)$. This follows from the fact that the normal bundle of $V \subset M$ respectively $W \subset M \times[0,1]$ is trivial.

More generally, let $X$ be a space and $\Phi: W \rightarrow X$ be a $B$-bordism between singular $B$-manifolds $\phi: M \rightarrow X$ and $\phi^{\prime}: M^{\prime} \rightarrow X$. If $f: X \rightarrow S^{1}$ represents an element $\alpha \in H^{1}(X, \mathbb{Z})$, then $f \circ \Phi$ is homotopic to a map $\Psi$ : $W \rightarrow S^{1}$ with $\Psi$ and $\Psi \mid \partial W$ being transverse to $1 \in S^{1}$ (moreover, the map $\Psi \mid \partial W$ with the corresponding properties may be given in advance). Then $\Psi^{-1}(1) \subset W$ is a $B$-bordism between the hypersurfaces $(\Psi)^{-1}(1) \cap M \subset M$ and $\Psi^{-1}(1) \cap M^{\prime} \subset M^{\prime}$, and restricting $\Phi$ to $\Psi^{-1}(1)$ now yields a singular $B$-bordism between singular hypersurfaces into $X$.

If $f^{\prime}: X \rightarrow S^{1}$ is homotopic to $f$, a similar construction as above gives a singular $B$-bordism between the resulting singular hypersurfaces into $X$.

Taken together, we obtain a homomorphism

$$
\cap: H^{1}(X, \mathbb{Z}) \times \Omega_{m}(X) \rightarrow \Omega_{m-1}(X) .
$$

The two theorems above now imply

4.4. Theorem. Let $X$ be a space and let $3 \leq m \leq 8$. Then (4.3) restricts to a homomorphism:

$$
H^{1}(X, \mathbb{Z}) \cap \Omega_{m}^{+}(X) \rightarrow \Omega_{m-1}^{+}(X)
$$

where $\Omega_{*}^{+}(X) \subset \Omega_{*}(X)$ is the subgroup of bordism classes which can be represented by singular manifolds which admit a metric with scal $>0$.

Proof. Theorem 4.2 implies that, given $f: M \rightarrow S^{1}$ (dual to a given class in $\left.H_{m-1}(M, \widetilde{o})\right)$ we find a homotopic map $g: M \rightarrow S^{1}$ which is transverse to 1 and such that the hypersurface $V=g^{-1}(1)$ is minimal for the $(m-1)$-volume (in dimension 8 we replace the given metric by one which is $C^{3}$-close). In any case, since the scalar curvature is continuous with respect to the $C^{3}$ norm on the space of all Riemannian metrics, $V$ is volume minimizing with respect to a metric with positive scalar curvature whenever we start with such a metric. By Theorem 4.1, it admits a metric with scal $>0$.

The spin version of (4.5) is used in Schick [15] to give a counterexample to the GLR-conjecture. We are now ready to use (4.5) to complete the proof of the theorem in the previous section.

\footnotetext{
${ }^{3}$ for example Spin bordism, oriented bordism or unoriented bordism
} 
Proof of Theorem 3.6. We use the same construction as in [15]: take a two dimensional singular bordism $f: F \rightarrow B(\mathbb{Z} / 3)^{2}$ which does not lie in $\Omega_{2}^{\text {spin, }+}\left(B(\mathbb{Z} / 3)^{2}\right)$ with the property that the image of its $K O$-orientation is zero in $\mathrm{KO}_{2}\left(B(\mathbb{Z} / 3)^{2}\right)$. We have already shown that such an $f$ exists (see 3.4). Then take the product with $i d: S^{1} \times S^{1} \times S^{1} \rightarrow B \mathbb{Z}^{3}$. The image of the $\mathrm{KO}$-orientation of the new singular bordism in $\mathrm{KO}_{5}(\mathrm{~B} \pi)$ is still zero. Doing surgeries we find a spin bordant manifold $M$ with fundamental group $\pi$ such that $[f \times i d]=[u: M \rightarrow B \pi]$ with $u: M \rightarrow B \pi$ being the classifying map of the universal covering. I.e. $M$ satisfies $\alpha(M)=0 \in K O_{5}(B \pi)$.

Now the Schoen-Yau method shows that $M$ cannot admit a metric with positive scalar curvature because taking successively minimal surfaces dual to the three canonical generators of $H^{1}\left(B \mathbb{Z}^{3}, \mathbb{Z}\right)$ otherwise would yield a manifold bordant to $F$ which would also admit a metric with scal $>0$. But this contradicts our choice of $F$.

\section{The example for non-orientable minimal sur- faces}

We want to show that (4.5) yields interesting results for non-orientable manifolds. If such a manifold has a metric with positive scalar curvature then the same is true for its orientation cover. The converse is not necessarily true. We will produce a five dimensional example whose orientation cover admits a metric with scal $>0$.

We start with the Klein bottle $K$, the non-orientable surface whose orientation cover is the torus. It follows from the $\mathrm{Wu}$ relations that $w_{2}(K)=$ $w_{1}(K)^{2}$. Hence the tangent bundle $T K$ admits a Pin(2)-structure (cf. [4]).

5.1. Lemma. If $W$ is an n-dimensional Pin(n)-manifold then its orientation cover $\widetilde{W}$ canonically carries a Spin $(n)$-structure.

Proof. If $P \rightarrow W$ is a $\operatorname{Pin}(n)$-principle bundle then $P \rightarrow P / \operatorname{Spin}(n)$ is a $\operatorname{Spin}(n)$-principle bundle over $P / \operatorname{Spin}(n)$, and $q: P / \operatorname{Spin}(n) \rightarrow W$ is a double covering classified by $w_{1}(W)$, i.e. we may regard $P / \operatorname{Spin}(n)$ as a model for $\widetilde{W}$. Moreover, if $\left(P, f: P \times_{\operatorname{Pin}(n)} \mathbb{R}^{n} \cong T W\right)$ represents a $\operatorname{Pin}(n)$ structure on $W$ then there is a canonical map $\tilde{f}: P \times_{\operatorname{Spin}(n)} \mathbb{R}^{n} \cong q^{*} T W=$ $T \widetilde{W}$ covering $f$. Hence $(P, \widetilde{f})$ represents a $\operatorname{Spin}(n)$-structure on $\widetilde{W}$.

It is an easy exercise to construct a $\operatorname{Pin}(2)$-structure on $K$ such that $\widetilde{K}=S^{1} \times S^{1}$ is the product of two copies of $S^{1}$ carrying the zero bordant $\operatorname{Spin}(1)$-structure. In the following we shall think of $K$ being equipped with 
this $\operatorname{Pin}(2)$-structure.

Next recall that $\pi_{1}(K)=\mathbb{Z} \ltimes \mathbb{Z}$ is the non-trivial semidirect product of $\mathbb{Z}$ with $\mathbb{Z}$, where the first factor is generated by the standard loop in $K$ with non-trivial normal bundle, and the second factor (which is the normal subgroup) by the loop with trivial normal bundle. The image of the fundamental group of the orientation cover is then $(2 \mathbb{Z}) \ltimes \mathbb{Z}=(2 \mathbb{Z}) \times \mathbb{Z}$. Let $p=\left(p_{1}, p_{2}\right): \pi_{1}(K) \rightarrow \mathbb{Z} / 2 \times \mathbb{Z} / 2$ be the projection corresponding to the structure of the semidirect product (i.e. the image of $p_{2}$ is generated by $p$ applied to the loop with trivial normal bundle, and $p_{1}$ represents the first Stiefel-Whitney class). Let us use the same letters for the corresponding maps

$$
p=\left(p_{1}, p_{2}\right): K \rightarrow B \mathbb{Z} / 2 \times B \mathbb{Z} / 2 .
$$

Recall also that a Pin(n)-structure on the tangent bundle of a manifold corresponds to a $P i n^{\prime}$-structure on its stable normal bundle (cf. [4]). In particular, $p_{2}: K \rightarrow B \mathbb{Z} / 2$ represents a bordism class $\left[p_{2}\right] \in \Omega_{2}^{p^{\prime \prime}}(B \mathbb{Z} / 2)$. Since the product of a $\operatorname{Pin}(n)$-manifold and a $\operatorname{Spin}(m)$-manifold canonically carries a $\operatorname{Pin}(n+m)$-structure, we can multiply $\left[p_{2}\right]$ with $\left[i d: S^{1} \rightarrow S^{1}\right]^{3} \in$ $\Omega_{3}^{\text {spin }}\left(B \mathbb{Z}^{3}\right)$, and we obtain $\left[p_{2} \times i d\right] \in \Omega_{5}^{p^{p i n^{\prime}}}\left(B \pi^{\prime}\right)$ with $\pi^{\prime}=\mathbb{Z} / 2 \times \mathbb{Z}^{3} \cdot p_{2} \times i d$ has a classifying map

$$
\left(\hat{\nu}_{N}, p_{2} \times i d\right): N=K \times\left(S^{1}\right)^{3} \longrightarrow B P i n^{\prime} \times B \pi^{\prime} .
$$

In $H^{1}\left(B\right.$ Pin $\left.^{\prime}, \mathbb{Z} / 2\right) \cong\left[B\right.$ Pin $\left.^{\prime}, B \mathbb{Z} / 2\right]$, let $w: B$ Pin $^{\prime} \rightarrow B \mathbb{Z} / 2$ represent the non-trivial element. Then $w \circ \hat{\nu}_{N}$ represents $w_{1}(N)$, i.e. $w \circ \hat{\nu}_{N}$ is homotopic to $p_{1} \circ p r_{K}: N \rightarrow K \rightarrow B \mathbb{Z} / 2$.

Now we can do surgeries on $N \times[0,1]$ to obtain a singular BPin'-bordism $\Phi: W \rightarrow B \pi^{\prime}$ between $\left(p_{2} \times i d\right)$ and a singular BPin'-manifold $\phi: M \rightarrow B \pi^{\prime}$ such that the classifying map

$$
\left(\hat{\nu}_{M}, \phi\right): M \longrightarrow B P \operatorname{Bin}^{\prime} \times B \pi^{\prime}
$$

is a 2-equivalence. Then $w \circ \hat{\nu}_{M}$ represents $w_{1}(M)$.

Note that since $\phi$ and $p_{2} \times i d$ are $p i n^{\prime}$-bordant so are $\left(\hat{\nu}_{M}, \phi\right)$ and $\left(\hat{\nu}_{N}, p_{2} \times\right.$ $i d$ ), and hence so are

$$
u=\left(w \circ \hat{\nu}_{M}, \phi\right): M \longrightarrow B \mathbb{Z} / 2 \times B \pi^{\prime}=B \pi, \quad \pi=\mathbb{Z} / 2 \times \pi^{\prime}
$$

and $\left(w \circ \hat{\nu}_{N}, p_{2} \times i d\right) \simeq\left(p_{1}, p_{2} \times i d\right)=(p \times i d)$, i.e. we have $[u]=[p \times i d] \in$ $\Omega_{*}^{p i n^{\prime}}(B \pi)$. 


\subsection{Proposition.}

(1) The orientation covering $\widetilde{M}$ does admit a metric with scal $>0$.

(2) $M$ does not admit a metric with scal $>0$.

Proof. (1) Since $\left(\hat{\nu}_{M}, \phi\right): M \rightarrow B P i n^{\prime} \times B \pi^{\prime}$ is a 2-equivalence it induces an isomorphism $\pi_{1}(M) \cong \mathbb{Z} / 2 \times \pi^{\prime}$. Therefore the map $u: M \longrightarrow B \pi$ above classifies the universal covering of $M$. Since $w \circ \hat{\nu}_{M}$ represents $w_{1}(M)$ the universal cover of the orientation covering $\widetilde{M}$ is classified by $\widetilde{u}=\phi \circ q$, where $q: \widetilde{M} \rightarrow M$ is the orientation covering. Using the bordism results of Gromov-Lawson mentioned in the introduction it follows that $\widetilde{M}$ has a metric with scal $>0$ if and only if $[\widetilde{u}] \in \Omega_{5}^{s p i n,+}\left(B \pi^{\prime}\right)$. However, using the orientation cover of the bordism $W$ between $M$ and $N$ and Lemma 5.1, $\widetilde{u}$ is spin bordant to

$$
S^{1} \times S^{1} \times\left(S^{1}\right)^{3} \rightarrow K \times\left(S^{1}\right)^{3} \stackrel{p_{2} \times i d}{\longrightarrow} B \mathbb{Z} / 2 \times B\left(\mathbb{Z}^{3}\right)=B \pi^{\prime} .
$$

This is zero bordant since we can fill in a disk $D^{2}$ into the first copy of the $S^{1}$ 's and still get a map to $B \pi^{\prime}$, i.e. $[\widetilde{u}]$ lies in the subgroup $\Omega_{5}^{s p i n,+}\left(B \pi^{\prime}\right)$.

(2) Consider the composition $u: M \rightarrow B \pi$ above and let us forget about the Pin(5)-structure of $M$. Then $u$ represents a bordism class $[u] \in \Omega_{5}^{o}(B \pi)$, the unoriented bordism group of $B \pi$. Moreover $p \times$ id : $N \rightarrow B \pi$ represents the same element. We now show that $[u]=[p \times i d] \notin \Omega_{5}^{o,+}(B \pi)$. To do this we cap the $\mathbb{Z} / 2$-orientation class with elements of $H^{1}(N, \mathbb{Z})$ which come from $B \pi^{\prime}$. Doing this three times with the generators of $H^{1}\left(B \mathbb{Z}^{3}, \mathbb{Z}\right) \subset$ $H^{1}\left(B \pi^{\prime}, \mathbb{Z}\right)$ eventually yields the bordism class of

$$
K \stackrel{p}{\rightarrow} B \mathbb{Z} / 2 \times B \mathbb{Z} / 2 \rightarrow B \pi .
$$

We have to show that this is not in $\Omega_{2}^{o,+}(B \pi)$. It suffices to show that $[p: K \rightarrow B \mathbb{Z} / 2 \times B \mathbb{Z} / 2]$ is not in $\Omega_{2}^{o,+}(B \mathbb{Z} / 2 \times B \mathbb{Z} / 2)$.

By the Gauss-Bonnet theorem, elements in $\Omega_{2}^{o,+}(X)$ for a space $X$ are represented by maps either from $S^{2}$ or $\mathbb{R} P^{2}$. Since $K=\mathbb{R} P^{2} \# \mathbb{R} P^{2}$ is null bordant, it is not bordant to $\mathbb{R} P^{2}$. Hence it remains to rule out that $p$ is bordant to some map $f: S^{2} \rightarrow B \mathbb{Z} / 2 \times B \mathbb{Z} / 2$. However, every map $S^{2} \rightarrow B \mathbb{Z} / 2 \times B \mathbb{Z} / 2$ is homotopically trivial and $S^{2}=\partial D^{2}$. I.e. it is enough to show that $[p] \neq 0 \in \Omega_{2}^{o}(B \mathbb{Z} / 2 \times B \mathbb{Z} / 2)$. To do so we compute a non-trivial characteristic number of $[p]$.

Let $H^{1}(B \mathbb{Z} / 2 \times B \mathbb{Z} / 2, \mathbb{Z} / 2)=\mathbb{Z} / 2\left\langle u_{1}\right\rangle \oplus \mathbb{Z} / 2\left\langle v_{1}\right\rangle$ be induced by the Künneth isomorphism. The two classes $u_{1}$ and $v_{1}$ pull back to two generators $p^{*} u_{1}$ and $p^{*} v_{1}$ in $H^{1}(K, \mathbb{Z} / 2)$. The latter are dual to the two standard 
loops generating the fundamental group. These intersect in exactly one point. Hence their intersection number is non-trivial, which implies that $<p^{*} u_{1} \cup p^{*} v_{1},[M]>\neq 0$. Hence we have found a non-trivial characteristic number of $[p]$, which now completes the proof, taking Theorem 4.4 into account.

\section{Twisted index of manifolds with universal cov- ering spin}

The Lichnerowicz method for positive scalar curvature obstructions a priori works only for spin manifolds. Rosenberg [11, 3.4] proved the following powerful version:

6.1. Theorem. If $M$ is an $n$-dimensional connected closed spin manifold with positive scalar curvature, then the index $\alpha(M)=0 \in K O_{n}\left(C_{\text {red }}^{*} \pi_{1}(M)\right)$.

In [9] Rosenberg explains how one can extend the theory to manifolds which are not necessarily spin, but where the universal covering is spin. This was later refined by Stephan Stolz to take non-orientable manifolds into account. The definite reference for these generalizations is [21].

Let $M$ be a connected manifold with fundamental group $\pi$. If the universal covering of $M$ is spin then the first two Stiefel-Whitney classes of $M$ pull back from two cohomology classes $u_{1} \in H^{1}(B \pi, \mathbb{Z} / 2)$ and $u_{2} \in H^{2}(B \pi, \mathbb{Z} / 2)$ via a map $u: M \rightarrow B \pi$ classifying the universal covering. $u_{1}$ can be interpreted as a homomorphism $\pi \rightarrow \mathbb{Z} / 2$ and $u_{2}$ determines (up to isomorphism) a central extension

$$
1 \longrightarrow \mathbb{Z} / 2 \longrightarrow \hat{\pi} \longrightarrow \pi \longrightarrow 1 \text {. }
$$

6.2. Definition. A supergroup $\gamma=(\pi, w, \hat{\pi})$ is a triple consisting of a group $\pi$, a homomorphism $w: \pi \rightarrow \mathbb{Z} / 2$ and a central extension as above.

Given a supergroup $\gamma$, Stolz constructs a corresponding bordism theory $\Omega_{*}(\gamma)$, a $C^{*}$-algebra $C_{r e d}^{*}(\gamma)$ and homomorphism $\alpha: \Omega_{*}(\gamma) \rightarrow K O_{*}\left(C_{r e d}^{*}(\gamma)\right)$. In the case where $w$ is trivial and the extension is split the constructions give back the homomorphism $\alpha: \Omega_{*}^{\text {spin }}(B \pi) \rightarrow K O_{*}\left(C_{\text {red }}^{*} \pi\right)$ used above. The bordism group is given by a (normal) $B$-bordism theory with $B=$ $B(\gamma)$ constructed from the supergroup data. Stolz then shows that a connected closed manifold $M$ determines a supergroup $\gamma(M)$ such that $M$ has a $B(\gamma(M))$-structure whose classifying map $M \rightarrow B(\gamma(M))$ is a 2-equivalence 
if $\operatorname{dim} M=n \geq 3 .{ }^{4}$ If $n \geq 5$ then it follows from the surgery results of Gromov-Lawson (cf. $[20,3.3])$ that $M$ has a positive scalar curvature metric if and only if $[M]:=\left[i d_{M}\right] \in \Omega_{n}(\gamma(M))^{+} \subset \Omega_{n}(\gamma(M))$, which actually motivated the definition of $\Omega_{*}(\gamma)$.

On the other hand we have the following generalization of Theorem 6.1:

6.3. Theorem. If $M$ has a metric with scal $>0$ then $\alpha([M])=0$.

Proof. This is a consequence of [21, 1.1 and 1.3]. As in the spin case it follows from an index theoretic description of the homomorphism $\alpha$ combined with a Lichnerowicz type argument.

We now show that the manifold $M$ defined in the previous section provides a non-spin counterexample to the generalized GLR-conjecture, which would say that a closed manifold $M$ of dimension $n \geq 5$ has a metric with scal $>0$ if and only if $\alpha([M])=0 \in K O_{n}\left(C_{\text {red }}^{*}(\gamma(M))\right)$.

First we need to work out the associated supergroup $\gamma(M)$. By definition $\pi=\pi_{1}(M)$ and $w=w_{1}(M)$. The definition of the extension follows from

6.4. Lemma. If $w_{2}(M)=u^{*}(e)$ for $u: M \rightarrow B \pi$ a map classifying the universal covering and $e \in H^{2}(B \pi, \mathbb{Z} / 2)$ then $\gamma(M)=\left(\pi, w_{1}(M), \hat{\pi}\right)$ with $\hat{\pi}$ an extension classified by $e$.

Proof. [21, $2.11(2)]$.

In our example we have $w_{2}(M)=u^{*}\left(u_{1}^{2}\right)$ using the notation introduced in the preceding section. It follows that

$$
\gamma(M)=\left(\pi=\mathbb{Z} / 2 \times \pi^{\prime}, p r_{1}: \pi \rightarrow \mathbb{Z} / 2, \operatorname{Pin}(1) \times \pi^{\prime}\right) .
$$

Going through the definitions we find $B(\gamma(M))=B P i n^{\prime} \times B \pi^{\prime}$. Hence we have an isomorphism $\Omega_{*}(\gamma(M)) \cong \Omega_{*}^{p i n^{\prime}}\left(B \pi^{\prime}\right)$.

On the other hand the corresponding $C^{*}$-algebra $C_{\text {red }}^{*}(\gamma(M))$ is given by

$$
C_{\text {red }}^{*}(\gamma(M)) \cong \mathcal{C} \ell_{1} \hat{\otimes} C_{\text {red }}^{*}\left(\pi^{\prime}\right)
$$

\footnotetext{
${ }^{4}$ Note that $B(\gamma(M))$ does not coincide with $B G(n, \gamma(M))$ as defined in $[21, \S 2]$ since we describe $\Omega_{*}(\gamma)$ through manifolds with a structure on its stable normal bundle. However, $M \rightarrow B(\gamma(M))$ is a 2-equivalence if and only if $M \rightarrow B G(n, \gamma(M))$ is one (for $n \geq 3$ ).
} 
where the tensor product we use here is the $C^{*}$-tensor product, which is uniquely defined in our case. To obtain this isomorphism we may regard $\gamma(M)$ as a product of supergroups $[21,2.4]$

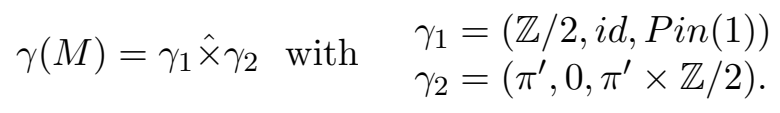

Then by $[21,8.2(2)$ and 8.3$]$ we obtain $C^{*}\left(\gamma_{1}\right) \cong \mathcal{C} \ell_{1}$ and $C^{*}\left(\gamma_{2}\right)=C_{\text {red }}^{*}\left(\pi^{\prime}\right)$, and $[21,8.4]$ then implies (6.5). Note that all the way through we deal with $\mathbb{Z} / 2$-graded $C^{*}$-algebras, where $C_{\text {red }}^{*}(\Gamma)$ for a group $\Gamma$ is trivially graded and $\mathcal{C} \ell_{1}$ has the standard grading.

6.6. Lemma. $K O_{5}\left(C_{r e d}^{*}(\gamma(M))\right) \cong \mathbb{Z}^{6}$.

Proof. First note that we deal with abelian groups. Therefore reduced and unreduced $C^{*}$-algebras coincide. Hence we have

$$
\begin{aligned}
K O_{n}\left(C_{r e d}^{*}(\gamma(M))\right) & \stackrel{\text { Def }}{=} K O_{0}\left(\mathcal{C} \ell_{n} \hat{\otimes} C_{r e d}^{*}(\gamma)\right) \\
& =K O_{0}\left(\mathcal{C} \ell_{n+1} \hat{\otimes} C^{*}\left(\mathbb{Z} / 2 \times \mathbb{Z}^{3}\right)\right)=K O_{n+1}\left(C^{*}\left(\mathbb{Z} / 2 \times \mathbb{Z}^{3}\right)\right) .
\end{aligned}
$$

For a discrete group $G$ we have (cf. [18, p. 14 and 1.5.4])

$$
K O_{n}\left(C_{\text {red }}^{*}(G \times \mathbb{Z})\right) \cong K O_{n}\left(C_{\text {red }}^{*}(G)\right) \oplus K O_{n-1}\left(C_{\text {red }}^{*}(G)\right) .
$$

Therefore the lemma now follows from the table below. We compute it using the fact that $\mathbb{Z} / 2$ has exactly two irreducible real representation, both of real type. Hence $K O_{*}(\mathbb{R} \mathbb{Z} / 2)=K O_{*}(\mathbb{R}) \oplus K O_{*}(\mathbb{R})$.

\begin{tabular}{l|l|l|l|l|l|l|l|l}
$n(\bmod 8)$ & 1 & 2 & 3 & 4 & 5 & 6 & 7 & 0 \\
\hline$K O_{n}\left(C^{*} \mathbb{Z} / 2\right)$ & $\mathbb{Z} / 2 \times \mathbb{Z} / 2$ & $\mathbb{Z} / 2 \times \mathbb{Z} / 2$ & 0 & $\mathbb{Z}^{2}$ & 0 & 0 & 0 & $\mathbb{Z}^{2}$
\end{tabular}

6.7. Corollary. The manifold $M$ defined in Section 5 has twisted index

$$
\alpha([M])=0 \in K O_{5}\left(C_{r e d}^{*}(\gamma(M))\right) .
$$

Proof. We have $\Omega_{*}(\gamma(M)) \cong \Omega_{*}^{p i n^{\prime}}\left(B \pi^{\prime}\right)$. Since the coefficients of Pin'bordism theory are completely 2 -torsion (cf. [4]) it follows from the AtiyahHirzebruch spectral sequence that $\Omega_{*}(\gamma(M))$ is completely 2-torsion. Hence $\alpha: \Omega_{5}(\gamma(M)) \rightarrow \mathrm{KO}_{5}\left(C_{\text {red }}^{*}(\gamma)\right)=\mathbb{Z}^{6}$ is trivial. 


\section{References}

[1] Baumslag, G., Dyer, E., and Heller, A.: "The topology of discrete groups", J. Pure Appl. Algebra 16, 1-47 (1980)

[2] Botvinnik, B. I., Gilkey, P., and Stolz, S.: "The Gromov-LawsonRosenberg Conjecture for groups with periodic cohomology", J. Diff. Geom. 46, 374-405 (1997)

[3] Charlap, L.S. and Vasquez, A.T.: Compact flat Riemannian manifolds II: The cohomology of $\mathbb{Z} /$ p-manifolds, Amer. J. Math. 87, 551-563 (1965)

[4] Giambalvo, V.: "Pin and Pin' cobordism", Proc. AMS 39, 395-401 (1973)

[5] Greenlees, J.P.C.: "K-homology of universal spaces and local cohomology of the representation ring", Topology 32, 295-308 (1993)

[6] Gromov, M. and Lawson, H.B.: "The classification of simply-connected manifolds of positive scalar curvature", Ann. of Math. 111, 423-434 (1980)

[7] Higson, N.: "Bivariant K-theory and the Novikov conjecture", preprint, Penn State; http://www.math.psu.edu/higson/research.html (1999)

[8] Morgan, F.: "Geometric measure theory, a beginner's guide", Academic Press (1988)

[9] Rosenberg, J.: " $C^{*}$-algebras, positive scalar curvature, and the Novikov conjecture", Publ. Math. IHES 58, 197-212 (1983)

[10] Rosenberg, J.: " $C^{*}$-algebras, positive scalar curvature, and the Novikov conjecture II", in: Araki, H. and Effros, E.G. (eds.), Geometric methods in operator algebra, vol. 123 of Pitman Research Notes in Math., 341-374, Longman/Wiley (1986)

[11] Rosenberg, J.: " $C^{*}$-algebras, positive scalar curvature, and the Novikov conjecture III", Topology 25, 319-336 (1986)

[12] Rosenberg, J. and Stolz, S.: "Manifolds of positive scalar curvature", in: Carlsson, G.E. et.al. (eds.): Algebraic topology and its applications, vol. 27 of M.S.R.I. publications, Springer, 241-267 (1994)

[13] Rosenberg, J. and Stolz, S.: "The "stable" version of the Gromov-Lawson conjecture", in: Proc. of the Cech Centennial Homotopy Theory Conference, vol. 181 of Contemporary Mathematics, 405-418 (1995)

[14] Smale, N.: "Generic regularity of homologically area minimizing hypersurfaces in ieght-dimensional manifolds", Comm. Anal. Geom. 1, 217-228 (1993)

[15] Schick, T.: "A counterexample to the (unstable) Gromov-Lawson-Rosenberg conjecture", Topology 37, 1165-1168 (1998)

[16] Schoen, R. and Yau, S.-T.: "Existence of incompressible minimal surfaces and the topology of three dimensional manifolds with non-negative scalar curvature", Ann. of Math. 110, 127-142 (1979) 
[17] Schoen, R. and Yau, S.T.: "On the structure of manifolds with positive scalar curvature", manuscr. mathem. 28, 159-183 (1979)

[18] Schröder, H.: "K-theory for real $C^{*}$-algebras and its applications", vol. 290 of Pitman Research Notes in Mathematics, Longman Scientific \& and Technical (1993)

[19] Stolz, S.: "Simply connected manifolds of positive scalar curvature", Ann. of Math. 136, 511-540 (1992)

[20] Stolz, S.: "Positive scalar curvature metrics-existence and classification", in: Proc. of the Intern. Congress of Math., Zürich, Switzerland 1994, Volume I, Birkhäuser, 626-636 (1995)

[21] Stolz, S.: "Concordance classes of positive scalar curvature", preprint 